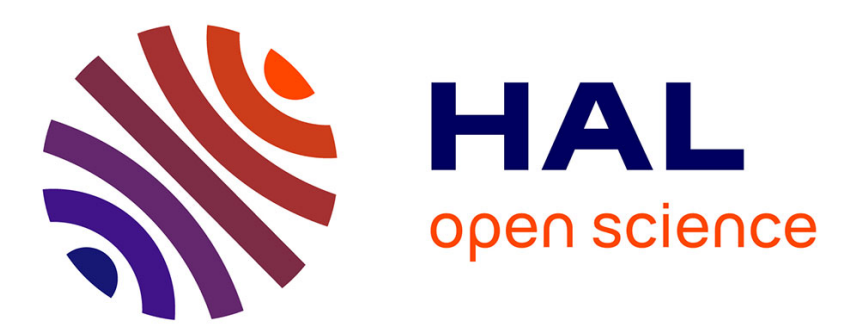

\title{
Dual Space of a Lattice as the Completion of a Pervin Space
}

Jean-Eric Pin

\section{To cite this version:}

Jean-Eric Pin. Dual Space of a Lattice as the Completion of a Pervin Space. Peter Höfner; Damien Pous; Georg Struth. Relational and Algebraic Methods in Computer Science, 10226, Springer, pp.2440, 2017, Lecture Notes in Computer Science, 10.1007/978-3-319-57418-9_2 . hal-02104104

\section{HAL Id: hal-02104104 https://hal.science/hal-02104104}

Submitted on 19 Apr 2019

HAL is a multi-disciplinary open access archive for the deposit and dissemination of scientific research documents, whether they are published or not. The documents may come from teaching and research institutions in France or abroad, or from public or private research centers.
L'archive ouverte pluridisciplinaire HAL, est destinée au dépôt et à la diffusion de documents scientifiques de niveau recherche, publiés ou non, émanant des établissements d'enseignement et de recherche français ou étrangers, des laboratoires publics ou privés. 


\title{
Dual space of a lattice as the completion of a Pervin space
}

\author{
Extended abstract
}

Jean-Éric Pin ${ }^{1}$

November 20, 2017

We assume the reader is familiar with basic topology on the one hand and finite automata theory on the other hand. No proofs are given in this extended abstract.

\section{Introduction}

The original motivation of this paper, as presented in [15], was to compute the dual space of a lattice of subsets of some free monoid $A^{*}$. According to StonePriestley duality, the dual space of a lattice can be identified with the set of its prime filters, but it is not always the simplest way to describe it. Consider for instance the Boolean algebra generated by the sets of the form $u A^{*}$, where $u$ is a word. Its dual space is equal to the completion of $A^{*}$ for the prefix metric and it can be easily identified with the set of finite or infinite words on $A$, a more intuitive description than prime filters.

Elaborating on this idea, one may wonder whether the dual space of a given lattice of subsets of a space can always be viewed as a completion of some sort. The answer to this question is positive and known for a long time: for Boolean algebras, the solution is detailed as an exercise in Bourbaki [7, Exercise 12, p. 211]. In the lattice case, the appropriate setting for this question is a very special type of spaces, the so-called Pervin spaces, which form the topic of this paper.

A Pervin space is a set $X$ equipped with a set of subsets, called the blocks of the Pervin space. Blocks are closed under finite intersections and finite unions and hence form a lattice of subsets of $X$. Pervin spaces are thus easier to define than topological spaces or (quasi)-uniform spaces. As a consequence, most of the standard topological notions, like convergence and cluster points, specialisation order, filters and Cauchy filters, complete spaces and completion are much easier to define for Pervin spaces.

The second motivation of this paper, also stemming from language theory, is the characterisation of classes of languages by inequations, which is briefly

\footnotetext{
${ }^{3}$ IRIF, CNRS and Université Paris-Diderot, Case 7014, 75205 Paris Cedex 13, France. Jean-Eric.Pin@irif.fr

* Funded by the European Research Council (ERC) under the European Unions Horizon 2020 research and innovation programme (grant agreement No 670624) and by the DeLTA project (ANR-16-CE40-0007)
} 
reviewed in Section 2. For regular languages on $A^{*}$, these inequations are of the form $u \leqslant v$ where $u$ and $v$ are elements of the free profinite monoid $\widehat{A^{*}}$. The main result of [14] states that any lattice of regular languages can be defined by a set (in general infinite) of such inequalities. The more general result of [15] states any lattice of languages (not necessarily regular) can be defined by a set of inequations of the form $u \leqslant v$, where $u$ and $v$ are now elements of $\beta A^{*}$, the Stone-Čech compactification of $A^{*}$.

It turns out that it is possible to give a simple proof of these two results using Pervin spaces. Let $\mathcal{L}$ be the set of blocks of a Pervin space $X$. Then the completion of $X$ can be defined as the set of valuations on $\mathcal{L}$. A valuation on $\mathcal{L}$ is simply a lattice morphism from $\mathcal{L}$ to the two-element Boolean algebra $\{0,1\}$. In particular, if $\mathcal{L}$ is the lattice of regular languages on $A^{*}$, then the completion of $A^{*}$ is $\widehat{A^{*}}$. If $\mathcal{L}$ is the lattice of all languages on $A^{*}$, then the completion of $A^{*}$ is $\beta A^{*}$.

Of course, valuations and prime ideals are just the same thing, but we prefer to use valuations, because they come with a very natural order relation: $v \leqslant w$ if and only if $v(L) \leqslant w(L)$ for all $L \in \mathcal{L}$. It is also natural to say that a set of blocks $\mathcal{K}$ satisfies the inequation $v \leqslant w$ if, for every $K \in \mathcal{K}, v(K) \leqslant w(K)$. Now, the characterisation of lattices by inequations takes the following form:

A set of blocks is a sublattice of $\mathcal{L}$ if and only if it can be defined by a set of inequations.

Taking for $\mathcal{L}$ the lattice of regular languages on $A^{*}$, one recovers the result of [14] and taking for $\mathcal{L}$ the lattice of all languages on $A^{*}$, one finds again the main result of [15]. Another result is worth mentioning. Let $\mathcal{L}$ be a lattice of subsets of $X$ and let $\mathcal{K}$ be a sublattice of $\mathcal{L}$. Then the following property holds:

The Pervin space $(X, \mathcal{L})$ is a subspace of the Pervin space $(X, \mathcal{K})$ and the completion of $(X, \mathcal{K})$ is a quotient of the completion of $(X, \mathcal{L})$.

Although this result looks like a contravariant property of duality theory, one has to be careful when defining a quotient space. This is fully discussed in Section 7 .

\section{Formal languages}

In this section, we briefly review the results on languages that motivated this paper. A lattice of languages is a set $\mathcal{L}$ of languages of $A^{*}$ containing $\emptyset$ and $A^{*}$ and closed under finite unions and finite intersections. It is closed under quotients ${ }^{1}$ if, for each $L \in \mathcal{L}$ and $u \in A^{*}$, the languages $u^{-1} L$ and $L u^{-1}$ are also in $\mathcal{L}$. A lattice of languages is a Boolean algebra if it is closed under complement.

An important object in this theory is the free profinite monoid $\widehat{A^{*}}$. It admits several equivalent descriptions, but we will only describe two of them. The reader is referred to $[4,5,29]$ for more details.

The free profinite monoid as the completion of a metric space.

A monoid $M$ separates two words $u$ and $v$ of $A^{*}$ if there exists a monoid morphism $\varphi: A^{*} \rightarrow M$ such that $\varphi(u) \neq \varphi(v)$. One can show that two distinct words can always be separated by a finite monoid.

\footnotetext{
${ }^{1}$ Recall that $u^{-1} L=\left\{x \in A^{*} \mid u x \in L\right\}$ and $L u^{-1}=\left\{x \in A^{*} \mid x u \in L\right\}$.
} 
Given two words $u, v \in A^{*}$, we set

$$
\begin{aligned}
& r(u, v)=\min \{|M| \mid M \text { is a monoid that separates } u \text { and } v\} \\
& d(u, v)=2^{-r(u, v)}
\end{aligned}
$$

with the usual conventions $\min \emptyset=+\infty$ and $2^{-\infty}=0$. Then $d$ is an ultrametric, that is, satisfies the following properties, for all $u, v, w \in A^{*}$,

(1) $d(u, v)=d(v, u)$,

(2) $d(u w, v w) \leqslant d(u, v)$ and $d(w u, w v) \leqslant d(u, v)$,

(3) $d(u, w) \leqslant \max \{d(u, v), d(v, w)\}$.

Thus $\left(A^{*}, d\right)$ is a metric space. Its completion, denoted by $\widehat{A^{*}}$, is called the free profinite monoid on $A$ and its elements are called profinite words. The term "monoid" needs to be justified. In fact, the multiplication on $A^{*}$ (the concatenation product) is uniformly continuous and hence can be extended in a unique way to a uniformly continuous operation on $\widehat{A^{*}}$. This operation makes $\widehat{A^{*}}$ a compact topological monoid. Recall that a topological monoid is a monoid $M$ equipped with a topology on $M$ such that the multiplication $(x, y) \rightarrow x y$ is a continuous map from $M \times M \rightarrow M$.

It is not so easy to give examples of profinite words which are not words, but here is one. In a compact monoid, the smallest closed subsemigroup containing a given element $x$ has a unique idempotent, denoted $x^{\omega}$. This is true in particular in a finite monoid and in the free profinite monoid. Thus if $x$ is a (profinite) word, so is $x^{\omega}$. Alternatively, one can define $x^{\omega}$ as the limit of the converging sequence $x^{n !}$. More details can be found in $[3,23]$.

\section{The free profinite monoid as a projective limit.}

Given a monoid morphism $f: A^{*} \rightarrow M$, we denote by $\sim_{f}$ the kernel congruence of $f$, defined on $A^{*}$ by $u \sim_{f} v$ if and only if $f(u)=f(v)$. For each pair of surjective morphisms $f: A^{*} \rightarrow M$ and $g: A^{*} \rightarrow N$ such that $\sim_{f} \subseteq \sim_{g}$, there is a unique surjective morphism $\pi_{f, g}: M \rightarrow N$ such that $g=\pi_{f, g} \circ f$. Moreover $\pi_{f, h}=\pi_{g, h} \circ \pi_{f, g}$ and $\pi_{f, f}=I d_{M}$.

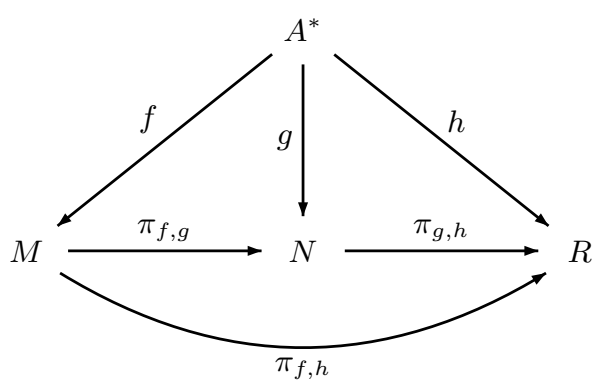

The monoid $\widehat{A^{*}}$ can be defined as the projective limit of the directed system formed by the surjective morphisms between finite $A$-generated monoids. A possible construction is to consider the compact monoid

$$
P=\prod_{f: A^{*} \rightarrow M_{f}} M_{f}
$$

where the product runs over all monoid morphisms $f$ from $A^{*}$ to some finite monoid $M_{f}$, equipped with the discrete topology. An element $\left(s_{f}\right)_{f: A^{*} \rightarrow M}$ of 
$P$ is compatible if $\pi_{f, g}\left(s_{f}\right)=s_{g}$. The set of compatible elements is a closed submonoid of $P$, which is equal to $\widehat{A^{*}}$.

We now come to profinite inequations. Let $u, v \in \widehat{A^{*}}$. A regular language $L$ of $A^{*}$ satisfies the inequation $u \leqslant v$ if the condition $u \in \bar{L}$ implies $v \in \bar{L}$, where $\bar{L}$ denotes the closure of $L$ in $\widehat{A^{*}}$. Here is the main result of [14]:

Proposition 2.1. Any lattice of regular languages can be defined by a set (in general infinite) of profinite inequalities.

This result is useful to analyse the expressive power of various fragments of monadic second order logic interpreted on finite words. It is of particular interest for lattices of regular languages closed under quotients. In this case the inequations can be directly interpreted in the ordered syntactic monoid. This notion was first introduced by Schützenberger in 1956 [25], but thereafter, he apparently only used the syntactic monoid.

Let $L$ be a language of $A^{*}$. The syntactic preorder of $L$ is the relation $\leqslant_{L}$ defined on $A^{*}$ by $u \leqslant_{L} v$ if and only if, for every $x, y \in A^{*}$,

$$
x u y \in L \Longrightarrow x v y \in L .
$$

The syntactic congruence of $L$ is the associated equivalence relation $\sim_{L}$, defined by $u \sim_{L} v$ if and only if $u \leqslant_{L} v$ and $v \leqslant_{L} u$.

The syntactic monoid of $L$ is the quotient $M(L)$ of $A^{*}$ by $\sim_{L}$ and the natural morphism $\eta_{L}: A^{*} \rightarrow A^{*} / \sim_{L}$ is called the syntactic morphism of $L$. The syntactic preorder $\leqslant_{L}$ induces an order on the quotient monoid $M(L)$. The resulting ordered monoid is called the syntactic ordered monoid of $L$. The syntactic morphism admits a unique continuous extension $\widehat{\eta}: \widehat{A^{*}} \rightarrow M$. For instance, if $\eta(u)=x$, then $\widehat{\eta}\left(u^{\omega}\right)=x^{\omega}$, where $x^{\omega}$ is the unique idempotent power of $x$ in $M$.

For instance, if $L$ is the language $\{a, a b a\}$, its syntactic monoid is the monoid $M=\{1, a, b, a b, b a, a b a, 0\}$ presented by the relations $a^{2}=b^{2}=b a b=0$. Its syntactic order is $0<a b<1,0<b a<1,0<a b a<a, 0<b$.

Let $\mathcal{L}$ be a lattice of regular languages closed under quotients. One can show that $\mathcal{L}$ satisfies the profinite inequation $u \leqslant v$ if and only if, for each $L \in \mathcal{L}$, $\eta_{L}(u) \leqslant \eta_{L}(v)$. This allows one to characterise the languages of $\mathcal{L}$ by a property of their ordered syntactic monoid. Here are three examples of such results, but many more can be found in the literature [23, 24].

(1) A regular language is finite if and only if its ordered syntactic monoid satisfies the inequations $y x^{\omega}=x^{\omega}=x^{\omega} y$ and $x^{\omega} \leqslant y$ for all profinite words $x \in \widehat{A^{*}}-\{1\}$ and $y \in \widehat{A^{*}}$.

(2) A famous result of Schützenberger [26] states that a regular language is star-free if and only if its syntactic monoid satisfies the equations $x x^{\omega}=x^{\omega}$ for all profinite words $x \in \widehat{A^{*}}$.

(3) Our third example is related to Boolean circuits. Recall that $\mathrm{AC}^{0}$ is the set of unbounded fan-in, polynomial size, constant-depth Boolean circuits. One can show $[6,27,28]$ that a regular language is recognised by a circuit in $\mathrm{AC}^{0}$ if and only if its syntactic monoid satisfies the equations $\left(x^{\omega-1} y\right)^{\omega}=$ $\left(x^{\omega-1} y\right)^{\omega+1}$ for all words $x$ and $y$ of the same length.

It is also possible to give an inequational characterisation of lattices of languages that are not regular [15]. The price to pay is to replace the profinite monoid by 
an even larger space, the Stone-Čech compactification of $A^{*}$, usually denoted $\beta A^{*}$. One can define $\beta A^{*}$ as the set of ultrafilters on the discrete space $A^{*}$. A second way to define it is to take the closure of the image of $A^{*}$ in the product space $\prod K$ where the product runs over all maps from $A^{*}$ into a compact Hausdorff space $K$ whose underlying set is $\mathcal{P}\left(\mathcal{P}\left(A^{*}\right)\right)$. Both spaces $\widehat{A^{*}}$ and $\beta A^{*}$ are compact, but only $\widehat{A^{*}}$ is a compact monoid.

Let $u, v \in \beta A^{*}$. We say that $L$ satisfies the ultrafilter inequality $u \rightarrow v$ if $u \in \bar{L}$ implies $v \in \bar{L}$, where $\bar{L}$ now denotes the closure of $L$ in $\beta A^{*}$. The main result of $[15]$ can be stated as follows:

Proposition 2.2. Any lattice of languages can be defined by a set (in general infinite) of ultrafilter inequalities.

In section 8 , we will recover Propositions 2.1 and 2.2 as a special case of Theorem 8.1. See also [13] for a duality point of view of these results.

\section{Pervin spaces}

It is time to introduce the main topic of this article. Let $X$ be a set. A lattice of subsets of $X$ is a subset of $\mathcal{P}(X)$ containing $\emptyset$ and $X$ and closed under finite intersections and finite unions. A Boolean algebra of subsets of $X$ is a lattice of subsets of $X$ closed under complement.

Given a lattice $\mathcal{L}$ of subsets of $X$, we denote by $\mathcal{L}^{s}$ the Boolean algebra generated by $\mathcal{L}$. There is a simple description of $\mathcal{L}^{s}$ using the set

$$
D(\mathcal{L})=\left\{L_{1}-L_{0} \mid L_{0}, L_{1} \in \mathcal{L}\right\} .
$$

of differences of members of $\mathcal{L}$. Indeed, Hausdorff [16] has shown that the Boolean algebra $\mathcal{L}^{s}$ consists of the finite unions of elements of $D(\mathcal{L})$.

\subsection{The category of Pervin spaces}

A Pervin structure on a set $X$ is a lattice $\mathcal{L}$ of subsets of $X$. The elements of $\mathcal{L}$ are called the blocks of the Pervin structure. A Pervin space is a set endowed with a Pervin structure. More formally, a Pervin space is a pair $(X, \mathcal{L})$ where $\mathcal{L}$ is a lattice of subsets of $X$. A Boolean Pervin space is a Pervin space in which $\mathcal{L}$ is a Boolean algebra.

Let $(X, \mathcal{K})$ and $(Y, \mathcal{L})$ be two Pervin spaces. A map $\varphi: X \rightarrow Y$ is said to be morphism if, for each $L \in \mathcal{L}, \varphi^{-1}(L) \in \mathcal{K}$. In other words, a map is a morphism if the preimage of a block is a block. It is readily seen that the composition of two morphisms is again a morphism and that the identity function on $X$ is a morphism. Pervin spaces together with their morphisms form the category Pervin of Pervin spaces.

Two Pervin spaces, both defined on the two-element set $\{0,1\}$, play an important role in this theory. The first one, the Boolean space $\mathbb{B}$, is defined by the lattice of all subsets of $\{0,1\}$. The second one, the Sierpinski space $\mathbb{S}$, is defined by the lattice $\{\emptyset,\{1\},\{0,1\}\}$. Note that the identity on $\{0,1\}$ is a morphism from $\mathbb{B}$ to $\mathbb{S}$ but it is not a morphism from $\mathbb{S}$ to $\mathbb{B}$. More examples of Pervin spaces are given in Section 5. 


\subsection{Pervin spaces as preordered sets}

A Pervin space $(X, \mathcal{L})$ is naturally equipped with a preorder $\leqslant \mathcal{L}$ on $X$ defined by $x \leqslant \mathcal{L} y$ if, for each $L \in \mathcal{L}$,

$$
x \in L \Longrightarrow y \in L .
$$

The associated equivalence relation $\sim_{\mathcal{L}}$ is defined on $X$ by $x \sim_{\mathcal{L}} y$ if, for each $L \in \mathcal{L}$

$$
x \in L \Longleftrightarrow y \in L .
$$

When the lattice $\mathcal{L}$ is understood, we will drop the index $\mathcal{L}$ and simply denote by $\leqslant$ and $\sim$ the preorder on $(X, \mathcal{L})$ and its associated equivalence relation. For instance, in the Boolean space, the preorder is the equality relation and in the Sierpiński space, the preorder is $0 \leqslant 1$.

It is easy to see that any morphism of Pervin spaces is order-preserving.

\subsection{Pervin spaces as topological spaces}

There are two topologies of interest on a Pervin space $(X, \mathcal{L})$. The first one, simply called the topology of $(X, \mathcal{L})$, is the topology based on the blocks of $\mathcal{L}$. The second one, called the symmetrical topology of $(X, \mathcal{L})$, is the topology based on the blocks of $\mathcal{L}^{s}$. In view of Hausdorff's result, these definitions can be summarized as follows:

Definition 3.1. The blocks of a Pervin space form a base of its topology. The differences of two blocks form a base of clopen sets of its symmetrical topology.

It follows immediately from the definition of the topology that the blocks containing a point $x$ form a basis of the filter $\mathcal{N}(x)$ of neighbourhoods of $x$ and that

$$
\mathcal{N}(x) \cap \mathcal{L}=\{L \in \mathcal{L} \mid x \in L\}
$$

The specialisation preorder, defined on any topological space $X$, is the relation $\leqslant$ defined on $X$ by $x \leqslant y$ if and only if $\overline{\{x\}} \subseteq\{y\}$ or, equivalently, if and only if $x \in \overline{\{y\}}$. It turns out that in a Pervin space $(X, \mathcal{L})$, the specialisation preorder coincides with the preorder $\leqslant \mathcal{L}$.

Recall that a topological space $X$ is a Kolmogorov space (or $T_{0}$-space) if for any two distinct points of $X$, there is an open set which contains one of these points and not the other. Kolmogorov Pervin spaces are easy to describe:

Proposition 3.1. Let $(X, \mathcal{L})$ be a Pervin space. The following conditions are equivalent:

(1) The preorder $\leqslant$ is a partial order,

(2) The relation $\sim$ is the equality relation,

(3) The space $(X, \mathcal{L})$ is Kolmogorov,

(4) The space $\left(X, \mathcal{L}^{s}\right)$ is Hausdorff.

Being Kolmogorov is a very desirable property for a Pervin space. Fortunately, it is easy to make a Pervin space Kolmogorov by taking its quotient by the relation $\sim$. That is, one considers the quotient space $X / \sim$ and one defines the 
blocks of $X / \sim$ to be the sets of the form $L / \sim$, for $L \in \mathcal{L}$. Slightly abusing notation, we set

$$
\mathcal{L} / \sim=\{L / \sim \mid L \in \mathcal{L}\}
$$

The Pervin space $(X / \sim, \mathcal{L} / \sim)$ is called the Kolmogorov quotient of $X$. Note that the natural map from $X$ to $X / \sim$ is a morphism of Pervin spaces which induces a lattice isomorphism from $\mathcal{L}$ to $\mathcal{L} / \sim$.

Compact Pervin spaces have some further interesting properties. First of all, blocks and compact open subsets are closely related. Note however that since our spaces are not necessarily Hausdorff, compact subsets are not necessarily closed.

Theorem 3.2. A compact open subset of a compact Pervin space is a block. If a Pervin space is compact for the symmetrical topology, then every block is compact open (for the usual topology). In particular, a subset of a compact Boolean Pervin space is a block if and only if it is compact open.

When $X$ is Kolmogorov and compact for the symmetrical topology, a few more characterisations of its blocks are available.

Theorem 3.3. Let $(X, \mathcal{L})$ be a Kolmogorov Pervin space that is compact for the symmetrical topology and let $L$ be a subset of $X$. Then the following conditions are equivalent:

(1) $L$ is a block of $\mathcal{L}$,

(2) $L$ is compact open in $(X, \mathcal{L})$,

(3) $L$ is an upset in $(X, \mathcal{L})$ and is clopen in $\left(X, \mathcal{L}^{s}\right)$,

(4) $L$ is an upset in $(X, \mathcal{L})$ and a block in $\left(X, \mathcal{L}^{s}\right)$.

\section{Complete Pervin spaces}

We have already seen that several topological definitions become much simpler in the case of a Pervin space. This is again the case for the notions studied in this section: Cauchy filters, complete spaces and completion.

Definition 4.1. A filter $\mathcal{F}$ on a Pervin space $X$ is Cauchy if and only if, for every block $L$, either $L \in \mathcal{F}$ or $L^{c} \in \mathcal{F}$.

One can show that it makes no difference to consider the symmetrical Pervin structure. More precisely, if $(X, \mathcal{L})$ is a Pervin space, then a filter on $X$ is Cauchy on $(X, \mathcal{L})$ if and only if it is Cauchy on $\left(X, \mathcal{L}^{s}\right)$. One can show, as in the case of a metric space, that a cluster point of a Cauchy filter is a limit point.

Definition 4.2. A Pervin space is complete if every Cauchy filter converges in the symmetrical topology.

Complete Pervin spaces admit the following characterisations.

Theorem 4.1. Let $(X, \mathcal{L})$ be a Pervin space. The following conditions are equivalent:

(1) $(X, \mathcal{L})$ is complete,

(2) $\left(X, \mathcal{L}^{s}\right)$ is complete, 
(3) $\left(X, \mathcal{L}^{s}\right)$ is compact.

If these conditions are satisfied, then $(X, \mathcal{L})$ is compact.

Note however that a compact Pervin space need not be complete as shown in Example 5.2. Just like in the case of a metric space, it is easy to describe the complete subspaces of a complete Pervin space.

Proposition 4.2. Every subspace of a complete Pervin space that is closed in the symmetrical topology is complete. A complete subspace of a Kolmogorov Pervin space is closed in the symmetrical topology.

We now come to the formal definition of the completion of a Pervin space.

Definition 4.3. A completion of a Pervin space $X$ is a complete Kolmogorov Pervin space $\widehat{X}$ together with a morphism $\imath: X \rightarrow \widehat{X}$ satisfying the following universal property: for each morphism $\varphi: X \rightarrow Y$, where $Y$ is a complete Kolmogorov Pervin space, there exists a unique morphism $\widehat{\varphi}: \widehat{X} \rightarrow Y$ such that $\widehat{\varphi} \circ \imath=\varphi$.

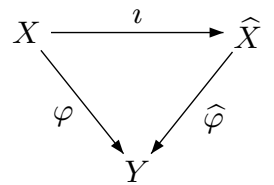

By standard categorical arguments, these conditions imply the unicity of the completion (up to isomorphism). The actual construction of the completion relies on the notion of valuation on a lattice.

Definition 4.4. A valuation on a lattice $\mathcal{L}$ is a lattice morphism from $\mathcal{L}$ into the Boolean lattice $\{0,1\}$.

In other words, a valuation is a function $v$ from $\mathcal{L}$ into $\{0,1\}$ satisfying the following properties, for all $L, L^{\prime} \in \mathcal{L}$ :

(1) $v(\emptyset)=0$ and $v(X)=1$,

(2) $v\left(L \cup L^{\prime}\right)=v(L)+v\left(L^{\prime}\right)$,

(3) $v\left(L \cap L^{\prime}\right)=v(L) v\left(L^{\prime}\right)$,

where the addition and the product denote the Boolean operations. Valuations are naturally ordered by setting $v \leqslant v^{\prime}$ if and only if $v(L) \leqslant v^{\prime}(L)$ for all $L \in \mathcal{L}$.

The completion of a Pervin space can now be constructed as follows. For each block $L$, let

$$
\widehat{L}=\{v \mid v \text { is a valuation on } \mathcal{L} \text { such that } v(L)=1\} .
$$

In particular, $\widehat{X}$ is the set of all valuations on $\mathcal{L}$ and one can show that the map $L \rightarrow \widehat{L}$ defines a lattice morphism from $\mathcal{L}$ to the lattice of subsets of $\widehat{X}$. Consequently, the set

$$
\widehat{\mathcal{L}}=\{\widehat{L} \mid L \in \mathcal{L}\}
$$

is a lattice and $(\widehat{X}, \widehat{\mathcal{L}})$ is a Pervin space. We now have a candidate for the completion, but we still need a candidate for the map $\imath: X \rightarrow \widehat{X}$. For each $x \in X$, we define $\imath(x)$ as the valuation on $\mathcal{L}$ such that $\imath(x)(L)=1$ if and only if $x \in L$. 
Theorem 4.3. The Pervin space $(\widehat{X}, \widehat{\mathcal{L}})$ is Kolmogorov and complete and the pair $(\imath,(\widehat{X}, \widehat{\mathcal{L}}))$ is the completion of $(X, \mathcal{L})$.

A Pervin space and its Kolmogorov quotient have isomorphic completions. Furthermore, completion and symmetrization are two commuting operations. More precisely, the symmetrical completion $\left(\widehat{X},(\widehat{\mathcal{L}})^{s}\right)$ of $(X, \mathcal{L})$ can also be obtained as the completion of $\left(X, \mathcal{L}^{s}\right)$.

A nice feature of completions is that they extend to morphisms.

Theorem 4.4. Let $\left(X, \mathcal{L}_{X}\right)$ and $\left(Y, \mathcal{L}_{Y}\right)$ be two Pervin spaces and let $\varphi: X \rightarrow$ $Y$ be a morphism.

(1) There exists a unique morphism $\widehat{\varphi}$ from $\left(\widehat{X}, \widehat{\mathcal{L}}_{X}\right)$ to $\left(\widehat{Y}, \widehat{\mathcal{L}}_{Y}\right)$ such that $\imath_{Y} \circ \varphi=\widehat{\varphi} \circ \imath_{X}$.

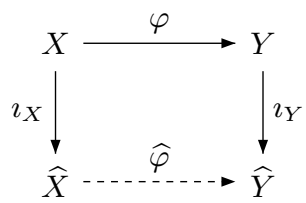

(2) The following formulas hold for all $v \in \widehat{X}$ and all $L \in \mathcal{L}_{Y}$ :

$$
\begin{aligned}
& \widehat{\varphi}(v)(L)=v\left(\varphi^{-1}(L)\right) \\
& \widehat{\varphi}^{-1}(\widehat{L})=\widehat{\varphi^{-1}(L)}
\end{aligned}
$$

Note also that in the category of Pervin spaces, completions preserve surjectivity, a property that does not hold for metric spaces. We now give a useful consequence of Theorem 4.4.

Corollary 4.5. Let $\varphi_{1}$ and $\varphi_{2}$ be two morphisms from $X$ to $Y$ and let $\widehat{\varphi}_{1}$ and $\widehat{\varphi}_{2}$ be their extensions from $\widehat{X}$ to $\widehat{Y}$. If $\varphi_{1} \leqslant \varphi_{2}$, then $\widehat{\varphi}_{1} \leqslant \widehat{\varphi}_{2}$.

The previous corollary is often used under a slightly different form, analogous to the Principle of extensions of identities of Bourbaki [7, Chapter I, Section 8.1, Corollary 1].

Corollary 4.6. Let $\varphi_{1}$ and $\varphi_{2}$ be two morphisms from $\left(\widehat{X}, \widehat{\mathcal{L}}_{X}\right)$ to $\left(\widehat{Y}, \widehat{\mathcal{L}}_{Y}\right)$. If, for all $x \in X, \varphi_{1}(x) \leqslant \varphi_{2}(x)$, then $\varphi_{1} \leqslant \varphi_{2}$. In particular, if $\varphi_{1}$ and $\varphi_{2}$ coincide on $X$, then they are necessarily equal.

\section{$5 \quad$ Examples of Pervin spaces}

In this series of examples, $(X, \mathcal{L})$ denotes a Pervin space.

Example 5.1 (Finite sets). Let $X=\mathbb{N}$ and $\mathcal{L}$ be the lattice formed by $X$ and the finite subsets of $X$. This space is Hausdorff but is neither compact nor complete. Indeed, the valuation $v$ given by $v(X)=1$ and $v(L)=0$ for each finite set $L$ defines a new element, denoted $-\infty$. The completion of $(X, \mathcal{L})$ is $(\widehat{X}, \widehat{\mathcal{L}})$, where $\widehat{X}=X \cup\{-\infty\}$ and $\widehat{\mathcal{L}}$ is the lattice formed by $\widehat{X}$ and the finite subsets of $X$. This lattice is isomorphic to $\mathcal{L}$. The order on $X$ is the equality relation, but in $\widehat{X}$, the order is given by $-\infty \leqslant x$ for each $x \in \widehat{X}$. 
Example 5.2 (Cofinite sets). Let $X=\mathbb{N}$ and let $\mathcal{L}$ be the lattice formed by the empty set and the cofinite subsets of $X$. This space is Kolmogorov and compact, but it is neither Hausdorff nor complete. Indeed, the valuation $v$ given by $v(L)=1$ for each cofinite set $L$ defines a new element, denoted $\infty$. The completion of $(X, \mathcal{L})$ is $(\widehat{X}, \widehat{\mathcal{L}})$, where $\widehat{X}=X \cup\{\infty\}$ and $\widehat{\mathcal{L}}$ is the lattice formed by the empty set and the cofinite subsets of $\widehat{X}$ containing $\infty$. This lattice is isomorphic to $\mathcal{L}$. The order on $X$ is the equality relation, but in $\widehat{X}$, the order is given by $x \leqslant \infty$ for each $x \in \widehat{X}$.

Example 5.3 (Finite or cofinite sets). Let $X=\mathbb{N}$ and let $\mathcal{L}$ be the Boolean algebra of all finite or cofinite subsets of $X$. This space is Hausdorff but it is neither compact nor complete. Indeed, the valuation $v$ given by $v(L)=1$ if $L$ is cofinite and $v(L)=0$ if $L$ is finite defines a new element, denoted $\infty$. The completion of $(X, \mathcal{L})$ is $(\widehat{X}, \widehat{\mathcal{L}})$, where $\widehat{X}=X \cup\{\infty\}$ and $\widehat{\mathcal{L}}$ is the Boolean algebra formed by the finite subsets of $X$ and by the cofinite subsets of $\widehat{X}$ containing $\infty$. This Boolean algebra is isomorphic to $\mathcal{L}$.

Example 5.4 (Finite sections). Let

$$
X=\left\{\frac{1}{n} \mid n \text { is a positive integer }\right\}
$$

and $\mathcal{L}$ be the lattice formed by $X$ and the subsets $L_{n}=\left\{\frac{1}{k} \mid 0<k \leqslant n\right\}$, for $n \geqslant 0$. This space is Kolmogorov and compact, but it is neither Hausdorff nor complete since the Cauchy filter $\mathcal{L}-\{\emptyset\}$ does not converge in $\left(X, \mathcal{L}^{s}\right)$. The valuation $v$ given by $v(X)=1$ and $v\left(L_{n}\right)=0$ for each $n$ defines a new element, denoted 0 . The completion of $(X, \mathcal{L})$ is $(\widehat{X}, \widehat{\mathcal{L}})$, where $\widehat{X}=X \cup\{0\}$ and $\widehat{\mathcal{L}}$ is the lattice formed by the empty set, $\widehat{X}$ and the finite subsets of $\widehat{X}$ containing 0 . This lattice is isomorphic to $\mathcal{L}$. The order on $\widehat{X}$ is the chain $0 \leqslant \cdots \leqslant \frac{1}{n} \leqslant \cdots \leqslant \frac{1}{2} \leqslant 1$. Every filter has 1 as a converging point. Indeed, if $L \in \mathcal{L}$ and $1 \in L$, then $L=X$ and $X$ is a member of all filters.

Example 5.5. Let $X=\{0,1,2\}$ and

$$
\mathcal{L}=\{\emptyset,\{1\},\{2\},\{1,2\},\{0,1,2\}\} .
$$

The preorder on $(X, \mathcal{L})$ is given by $0 \leqslant 1$ and $0 \leqslant 2$. Let $\mathcal{F}=\{\{1,2\},\{0,1,2\}\}$ be the filter generated by $\{1,2\}$. Then $\mathcal{F}$ is converging to 0 but it is not Cauchy since neither $\{1\}$ nor its complement are in $\mathcal{F}$.

Example 5.6. Let $X=\mathbb{N}$ and

$$
\mathcal{L}=\{\text { finite subsets of } \mathbb{N}\} \cup\left\{\{0\}^{c}\right\} .
$$

The preorder on $(X, \mathcal{L})$ is given by $0<n$ for each positive integer $n$. Let $\mathcal{F}$ be the Cauchy filter of all cofinite subsets of $\mathbb{N}$. Then 0 is the unique limit point of $\mathcal{F}$ in $(X, \mathcal{L})$ but $\mathcal{F}$ has no limit point in $\left(X, \mathcal{L}^{s}\right)$.

Example 5.7. Let $\left(X_{1}, \mathcal{L}_{1}\right)$ be the Pervin space considered in Example 5.1 and let $\left(X_{2}, \mathcal{L}_{2}\right)$ be the Pervin space considered in Example 5.4. Let $\varphi: X_{1} \rightarrow X_{2}$ be the map defined by $\varphi(n)=\frac{1}{n+1}$. Then $\varphi$ is a morphism and its completion $\widehat{\varphi}: \widehat{X}_{1} \rightarrow \widehat{X}_{2}$ is given by $\widehat{\varphi}(-\infty)=0$. 


\section{Duality results}

This section presents the links between Pervin spaces and duality theory. It relies on more advanced topological notions.

Duality theory provides three different representations of bounded distributive lattices via Priestley spaces, spectral spaces and pairwise Stone spaces $[8,10,12,17]$. Is it possible to recover these results using Pervin spaces? Well, not quite. Indeed, while duality is concerned with abstract distributive lattices, we only consider concrete ones, already given as a lattice of subsets. However, Pervin spaces allow one to recover these three representations for concrete distributive lattices. Let us first recall the definitions.

A topological space is zero-dimensional if it has a basis consisting of clopen subsets. It is totally disconnected if its connected components are singletons. It is well known that a compact space is zero-dimensional if and only if it is totally disconnected.

A Stone space is a compact totally disconnected Hausdorff space. A pairwise Stone space is a bitopological space $\left(X, \mathcal{T}_{1}, \mathcal{T}_{2}\right)$ which is pairwise compact, pairwise Hausdorff, and pairwise zero-dimensional.

A Priestley space is an ordered compact topological space $(X, \leqslant)$ satisfying the following separation property: if $x \notin y$, then there exists a clopen upset $U$ of $X$ such that $x \in U$ and $y \notin U$.

A subset $S$ of a topological space $X$ is irreducible if and only if, for each finite family $\left(F_{i}\right)_{i \in I}$ of closed sets, the condition $S \subseteq \bigcup_{i \in I} F_{i}$ implies that there exists $i \in I$ such that $S \subseteq F_{i}$. A topological space $X$ is sober if every irreducible closed subset of $X$ is the closure of exactly one point of $X$.

A topological space is spectral if it is Kolmogorov and sober and the set of its compact open subsets is closed under finite intersection and form a basis for its topology.

The relevance of Pervin spaces to duality theory is summarized in the following result:

Theorem 6.1. The completion of a Pervin space $(X, \mathcal{L})$ is the Stone dual of $\mathcal{L}$.

To complete this result, it just remains to requalify complete Pervin spaces as Priestley spaces, spectral spaces and pairwise Stone spaces.

Priestley spaces. If $(X, \mathcal{L})$ is a complete Pervin space, then $\left(X, \mathcal{L}, \leqslant_{\mathcal{L}}\right)$ is a Priestley space. The proof relies on the following variation on the prime filter theorem from order theory.

Proposition 6.2. Let $\mathcal{K}$ be a sublattice of a lattice $\mathcal{L}$ and let $L$ be an element of $\mathcal{L}-\mathcal{K}$. Then there exist two valuations $v_{0}$ and $v_{1}$ on $\mathcal{L}$ such that $v_{0}(L)=0$, $v_{1}(L)=1$ and $v_{1} \leqslant v_{0}$ on $\mathcal{K}$.

By the way, this result not only gives the separation property of Priestley spaces, but it is also of frequent use in the theory of Pervin spaces.

Spectral spaces. A compact Kolmogorov Pervin space need not be sober. However, if $(X, \mathcal{L})$ is a Kolmogorov Pervin space and if $\left(X, \mathcal{L}^{s}\right)$ is compact, then $(X, \mathcal{L})$ is spectral. In particular, a complete Pervin space is spectral. 
Pairwise Stone spaces. Let $\mathcal{L}^{c}=\left\{L^{c} \mid L \in \mathcal{L}\right\}$ be the set of complements of blocks of $X$. Then $\left(X, \mathcal{L}^{c}\right)$ is also a Pervin space and if $(X, \mathcal{L})$ is complete, then $\left(X, \mathcal{L}, \mathcal{L}^{c}\right)$ is a pairwise Stone space.

Let $(X, \mathcal{L})$ be a Pervin space and let $(\widehat{X}, \widehat{\mathcal{L}})$ be its completion. Then $\widehat{\mathcal{L}}$ is the set of all compact open subsets of $\widehat{X}$. It is also the set of upsets of $\widehat{X}$ that are clopen in the symmetrical topology. Moreover, the lattices $\mathcal{L}$ and $\widehat{\mathcal{L}}$ are isomorphic lattices.

These isomorphisms can be given explicitly. We just describe here the Kolmogorov case, which is simpler. Indeed, if $X$ is Kolmogorov, then the preorder on $X$ is an order and $\imath$ defines an embedding from $X$ into $\widehat{X}$. We tacitly make use of this embedding to identify $X$ with a subset of $\widehat{X}$.

Theorem 6.3. Let $(X, \mathcal{L})$ be a Kolmogorov Pervin space. Then the maps $L \mapsto \widehat{L}$ and $K \mapsto K \cap X$ are mutually inverse lattice isomorphisms between $\mathcal{L}$ and $\widehat{\mathcal{L}}$.

If $\mathcal{L}$ is a Boolean algebra, then all previous results simplify greatly. First, the preorders on $X$ and on $\widehat{X}$ are equivalence relations. Next, we have:

Proposition 6.4. Let $(X, \mathcal{L})$ be a Boolean Pervin space. Then $\mathcal{L}$ forms a basis of clopen sets. Furthermore, $X$ is Kolmogorov if and only if it is Hausdorff. Moreover, $(\widehat{X}, \widehat{\mathcal{L}})$ is a Hausdorff compact space and $\widehat{\mathcal{L}}$ is the Boolean algebra of clopen sets of $\widehat{X}$.

Furthermore, Theorem 6.3 can be restated as follows.

Theorem 6.5. Let $(X, \mathcal{L})$ be a Hausdorff Boolean Pervin space. Then the formulas $\widehat{L}=\bar{L}$ and $\bar{L} \cap X=L$ hold for all $L \in \mathcal{L}$. The maps $L \mapsto \bar{L}$ and $K \mapsto K \cap X$ are mutually inverse isomorphisms of Boolean algebra between $\mathcal{L}$ and $\widehat{\mathcal{L}}$.

In particular, the following formulas hold for all $L, L_{1}, L_{2} \in \mathcal{L}$ :

$$
\overline{L_{1} \cup L_{2}}=\bar{L}_{1} \cup \bar{L}_{2}, \quad \overline{L_{1} \cap L_{2}}=\bar{L}_{1} \cap \bar{L}_{2} \quad \text { and } \quad \overline{L^{c}}=\bar{L}^{c} \text {. }
$$

\section{Quotient spaces}

Let $(X, \mathcal{L})$ be a Pervin space and let $\mathcal{K}$ be a sublattice of $\mathcal{L}$. Denote by $\widehat{X}^{\mathcal{L}}$ the completion of $(X, \mathcal{L})$ and by $\widehat{X}^{\mathcal{K}}$ the completion of $(X, \mathcal{K})$. Then the following result holds.

Theorem 7.1. Let $\mathcal{L}$ be a lattice of subsets of $X$ and let $\mathcal{K}$ be a sublattice of $\mathcal{L}$. Then the identity function on $X$ is a morphism from $(X, \mathcal{L})$ to $(X, \mathcal{K})$ and its completion is a quotient map from $\widehat{X}^{\mathcal{L}}$ onto $\widehat{X}^{\mathcal{K}}$.

This theorem looks like an almost immediate consequence of Theorem 4.4 but there is a missing bit: we did not yet define the notion of a quotient map in the category of Pervin spaces.

A natural attempt would be to mimic the definition used for topological spaces (and for quasi-uniform spaces): a quotient map $\varphi: X \rightarrow Y$ should be a 
surjective morphism ${ }^{2}$ such that $Y$ is equipped with the final Pervin structure induced by $\varphi$. The second condition states that a subset $S$ of $Y$ is a block if and only if $\varphi^{-1}(S)$ is a block. However, Theorem 7.1 does not work under this definition. Indeed, we have already seen that the identity function $I$ on $\{0,1\}$ induces a morphism from the Boolean space $\mathbb{B}$ to the Sierpiński space $\mathbb{S}$. It is easy to see that these two spaces are isomorphic to their completion and that $\widehat{I}=I$. Consequently, $I$ should be a quotient map from $\mathbb{B}$ to $\mathbb{S}$. However, $\{0\}$ is a subset of $\mathbb{S}$ such that $I^{-1}(0)$ is a block of $\mathbb{B}$, but it is not a block of $\mathbb{S}$. Thus our definition of a quotient map has to be improved as follows:

Definition 7.1. Let $X$ and $Y$ be Pervin spaces. A surjective morphism $\varphi$ from $X$ to $Y$ is a quotient map if and only if each upset $U$ of $Y$ such that $\varphi^{-1}(U)$ is a block of $X$ is a block of $Y$.

Following [1], let Prost denote the category of preordered sets, with orderpreserving maps as morphisms. In the language of category theory, we viewed the category Pervin as a concrete category over the category Prost. That is, the forgetful functor now maps a Pervin space $(X, \mathcal{L})$ not to the set $X$, but to the preordered set $\left(X, \leqslant_{\mathcal{L}}\right)$. This definition can be rephrased in purely categorical terms, in which the role of the category Prost is even more apparent.

Proposition 7.2. Let $\varphi: X \rightarrow Y$ be a surjective morphism of Pervin spaces. The following conditions are equivalent:

(1) $\varphi$ is a quotient map,

(2) for each Pervin space $Z$, any preorder-preserving map $\psi: Y \rightarrow Z$ such that $\psi \circ \varphi$ is a morphism is a morphism,

(3) every preorder-preserving map $\psi$ from $Y$ to the Sierpinski space such that $\psi \circ \varphi$ is a morphism is also a morphism.

\section{Inequations}

We now give a general formulation of the results [14] and [15]. We first need an abstract definition of the notion of inequations.

Definition 8.1. Let $(X, \mathcal{L})$ be a Pervin space, let $L$ be a block of $X$ and let $(v, w)$ be a pair of valuations on $\mathcal{L}$. Then $L$ satisfies the inequation $v \leqslant w$ if $v(L) \leqslant w(L)$. More generally, a set of blocks $\mathcal{K}$ satisfies the inequation $v \leqslant w$ if, for every $K \in \mathcal{K}, v(K) \leqslant w(K)$.

Definition 8.2 can be easily extended to a set of inequalities as follows:

Definition 8.2. Given a set $S$ of inequations, a block $L$ satisfies $S$ if it satisfies all the inequations of $S$. Similarly, a set of blocks $\mathcal{K}$ satisfies $S$ if it satisfies all the inequations of $S$. Finally the set of all blocks of $X$ satisfying $S$ is called the set of blocks defined by $S$.

Formally, an inequation is thus a pair $(v, w)$ of valuations on $\mathcal{L}$. We are now ready to state the main result of this section.

\footnotetext{
${ }^{2}$ Formally, an epimorphism, but it is easy to see that in the category Pervin epimorphisms coincide with surjective morphisms.
} 
Theorem 8.1. Let $(X, \mathcal{L})$ be a Pervin space. A set of blocks of $X$ is a sublattice of $\mathcal{L}$ if and only if it can be defined by a set of inequations.

Back to languages. Let $X=A^{*}$ and let $\operatorname{Reg}\left(A^{*}\right)$ be the Boolean algebra of all regular languages on $A$. Almeida [2] has proved that the dual space of this Boolean algebra is the profinite monoid $\widehat{A^{*}}$. In other words, $\widehat{A^{*}}$ is the completion of the Pervin space $\left(A^{*}, \operatorname{Reg}\left(A^{*}\right)\right)$. This result can be briefly explained as follows. In one direction, each profinite word $v$ defines a valuation on $\operatorname{Reg}\left(A^{*}\right)$ defined by $v(L)=1$ if and only if $v \in \bar{L}$, where $\bar{L}$ denotes the closure of $L$ in $\widehat{A^{*}}$.

In the opposite direction, let $M$ be a finite monoid, $\varphi: A^{*} \rightarrow M$ be a monoid morphism and $v$ a valuation on $\operatorname{Reg}\left(A^{*}\right)$. Since $v\left(A^{*}\right)=1$ and $A^{*}=$ $\bigcup_{m \in M} \varphi^{-1}(m)$, one gets

$$
1=v\left(A^{*}\right)=\sum_{m \in M} v\left(\varphi^{-1}(m)\right)
$$

Consequently, there exists an $m \in M$ such that $v\left(\varphi^{-1}(m)\right)=1$. This $m$ is unique since if $v\left(\varphi^{-1}\left(m^{\prime}\right)\right)=1$ for some $m^{\prime} \neq m$, then, as $\varphi^{-1}(m) \cap \varphi^{-1}(m)=\emptyset$, one gets

$$
v(\emptyset)=v\left(\varphi^{-1}(m) \cap \varphi^{-1}(m)\right)=v\left(\varphi^{-1}(m)\right) v\left(\varphi^{-1}\left(m^{\prime}\right)\right)=1,
$$

a contradiction. Therefore, if $v$ is a valuation on $\operatorname{Reg}\left(A^{*}\right)$, there exists a unique profinite word $u$ such that, for each monoid morphism $\varphi: A^{*} \rightarrow M, \widehat{\varphi}(u)$ is the unique element $m \in M$ such that $v\left(\varphi^{-1}(m)\right)=1$.

Since $\widehat{A^{*}}$ is the completion of the Pervin space $\left(A^{*}, \operatorname{Reg}\left(A^{*}\right)\right)$, a direct application of Theorem 8.1 gives back Proposition 2.1.

To recover Proposition 2.2, let us first recall that $\beta A^{*}$ is the set of ultrafilters of the Boolean algebra $\mathcal{P}\left(A^{*}\right)$. Since, in the Boolean case, valuations and ultrafilters are essentially the same thing ${ }^{3}$, the completion of the Pervin space $\left(A^{*}, \mathcal{P}\left(A^{*}\right)\right)$ is isomorphic to $\beta A^{*}$ and Theorem 8.1 gives back Proposition 2.2.

\section{$9 \quad$ Bibliographic notes}

Pervin spaces were originally introduced by Pervin [22] to prove that every topological space can be derived from a quasi-uniform space. Since then, they have been regularly used to provide examples or counterexamples on quasi-uniform spaces but surprisingly, only two short articles seem to have been specifically devoted to their study, one by Levine in 1969 [21] and another one by Császár in 1993 [9]. In fact, Pervin spaces are so specific that their properties mostly appear in the literature as corollaries of more general results on quasi-uniform spaces. For instance, a quasi-uniform space is isomorphic to a Pervin space if and only if it is transitive and totally bounded. The reader interested in quasi-uniform spaces is refereed to the remarkable surveys written by Künzi [18, 19, 20]. Most notions introduced in this paper are actually adapted from the corresponding

\footnotetext{
${ }^{3}$ Let us define the characteristic function of an ultrafilter $\mathcal{U}$ as the map from $\mathcal{P}\left(A^{*}\right)$ to $\{0,1\}$ taking value 1 on $\mathcal{U}$ and 0 elsewhere. It is easy to see that it is a valuation on $\mathcal{P}\left(A^{*}\right)$. Conversely, if $v$ is a valuation on $\mathcal{P}\left(A^{*}\right)$, then $v^{-1}(1)$ is an ultrafilter.
} 
notions on quasi-uniform spaces, but they often become much simpler in the context of Pervin spaces.

Finally, the inspiring article of Erné [11], which sheds additional light on Pervin spaces, is highly recommended.

\section{Conclusion}

As we explained in the introduction, our original motivation was to find a simple way to describe the dual space of a lattice of subsets by a suitable completion. Metric spaces did not cover our needs, even in the case of Boolean algebras, except in the case of countable Boolean algebras. Uniform spaces did not suffice when dealing with lattices. Quasi-uniform spaces, on the other hand, while fulfilling our requirements, seemed to be too general a tool for our purpose. However, we soon realised that we only needed a very special class of quasiuniform spaces, the Pervin spaces. To our surprise, turning to Pervin spaces did not only simplify a number of results and proofs, but also lead us to an other point of view on Stone's duality. Moreover, it led us to a notion of quotient space which seems to be more appropriate in the ordered case.

\section{Acknowledgements}

I would like to thank Mai Gehrke and Serge Grigorieff for many fruitful discussions on Pervin spaces. I would also like to thank Daniela Petrişan for her critical help on categorical notions used in this paper. Encouragements from Hans-Peter A. Künzi and Marcel Erné were greatly appreciated.

\section{References}

[1] J. AdÁmek, H. Herrlich and G. E. Strecker, Abstract and concrete categories: the joy of cats, Repr. Theory Appl. Categ. ,17 (2006), 1-507. Reprint of the 1990 original [Wiley, New York; MR1051419].

[2] J. Almeida, Residually finite congruences and quasi-regular subsets in uniform algebras, Portugalice Mathematica 46 (1989), 313-328.

[3] J. Almeida, Finite semigroups and universal algebra, World Scientific Publishing Co. Inc., River Edge, NJ, 1994. Translated from the 1992 Portuguese original and revised by the author.

[4] J. Almeida, Profinite semigroups and applications, in Structural theory of automata, semigroups and universal algebra, V. B. Kudryavtsev and I. G. Rosenberg (eds.), New York, 2005, pp. 1-45, Springer.

[5] J. Almeida And P. Weil, Relatively free profinite monoids: an introduction and examples, in NATO Advanced Study Institute Semigroups, Formal Languages and Groups, J. Fountain (ed.), vol. 466, pp. 73-117, Kluwer Academic Publishers, 1995.

[6] D. A. M. Barrington, K. Compton, H. Straubing and D. Thérien, Regular languages in $\mathrm{NC}^{1}$, J. Comput. System Sci. 44,3 (1992), 478-499. 
[7] N. Bourbaki, General topology. Chapters 1-4, Elements of Mathematics (Berlin), Springer-Verlag, Berlin, 1998.

[8] D. M. Clark And B. A. Davey, Natural dualities for the working algebraist, Cambridge Studies in Advanced Mathematics vol. 57, Cambridge University Press, Cambridge, 1998.

[9] A. CsászÁr, D-completions of Pervin-type quasi-uniformities, Acta Sci. Math. 57,1-4 (1993), 329-335.

[10] B. A. Davey And H. A. Priestley, Introduction to Lattices and Order, 2nd edition, Cambridge University Press, 2002.

[11] M. ERnÉ, Ideal completions and compactifications, Appl. Categ. Structures $\mathbf{9 , 3}(2001), 217-243$.

[12] M. GEHRKe, Canonical extensions, Esakia spaces, and universal models, in Leo Esakia on duality in modal and intuitionistic logics, pp. 9-41, Outst. Contrib. Log. vol. 4, Springer, Dordrecht, 2014.

[13] M. Gehrke, Stone duality, topological algebra, and recognition, J. Pure Appl. Algebra 220,7 (2016), 2711-2747.

[14] M. Gehrke, S. Grigorieff And J.-E. Pin, Duality and equational theory of regular languages, in ICALP 2008, Part II, L. Aceto and al. (eds.), Berlin, 2008, pp. 246-257, Lect. Notes Comp. Sci. vol. 5126, Springer.

[15] M. Gehrke, S. Grigorieff And J.-E. Pin, Duality and equational theory of regular languages, in ICALP 2010, Part II, S. e. a. Abramsky (ed.), Berlin, 2010, pp. 151-162, Lect. Notes Comp. Sci. vol. 6199, Springer.

[16] F. Hausdorff, Set theory, Chelsea Publishing Company, New York, 1957. Translated by John R. Aumann, et al.

[17] P. T. Johnstone, Stone spaces, Cambridge Studies in Advanced Mathematics vol. 3, Cambridge University Press, Cambridge, 1986. Reprint of the 1982 edition.

[18] H.-P. A. KünZI, Quasi-uniform spaces in the year 2001, in Recent progress in general topology, II, pp. 313-344, North-Holland, Amsterdam, 2002.

[19] H.-P. A. KüNZI, Uniform structures in the beginning of the third millenium, Topology Appl. 154,14 (2007), 2745-2756.

[20] H.-P. A. KüNZI, An introduction to quasi-uniform spaces, in Beyond topology, pp. 239-304, Contemp. Math. vol. 486, Amer. Math. Soc., Providence, RI, 2009.

[21] N. Levine, On Pervin's quasi uniformity, Math. J. Okayama Univ. 14 $(1969 / 70), 97-102$.

[22] W. J. Pervin, Quasi-uniformization of topological spaces, Math. Ann. 147 (1962), 316-317. 
[23] J.-É. PIN, Profinite methods in automata theory, in 26th International Symposium on Theoretical Aspects of Computer Science (STACS 2009), S. Albers and J.-Y. Marion (eds.), pp. 31-50, Internationales Begegnungsund Forschungszentrum für Informatik (IBFI), Schloss Dagstuhl, Germany, 2009.

[24] J.-É. Pin, Equational descriptions of languages, Int. J. Found. Comput. S. 23 (2012), 1227-1240.

[25] M.-P. Schützenberger, Une théorie algébrique du codage, in Séminaire Dubreil-Pisot, année 1955-56, Exposé No. 15, 27 février 1956, 24 pages, Inst. H. Poincaré, Paris, 1956. http://igm.univ-mlv.fr/ berstel/Mps/Travaux/ A/1956CodageSemDubreil.pdf.

[26] M.-P. SchÜtzenberger, On finite monoids having only trivial subgroups, Information and Control 8 (1965), 190-194.

[27] H. Straubing, Finite automata, formal logic, and circuit complexity, Progress in Theoretical Computer Science, Birkhäuser Boston Inc., Boston, MA, 1994.

[28] H. Straubing, On logical descriptions of regular languages, in LATIN 2002, Berlin, 2002, pp. 528-538, Lect. Notes Comp. Sci. n²286, Springer.

[29] P. WeIL, Profinite methods in semigroup theory, Int. J. Alg. Comput. 12 (2002), 137-178. 